\title{
DEPENDENCIA HIDROLÓGICA Y REGULATORIA EN LA FORMACIÓN DE PRECIO DE LA ENERGÍA EN UN SISTEMA HIDRODOMINADO: CASO SISTEMA ELÉCTRICO COLOMBIANO
}

\author{
María del Carmen Quintero Quintero* \\ Felipe Isaza Cuervo**
}

Recibido: $14 / 12 / 2012$

Aceptado: 07/05/2013

\section{RESUMEN}

Este trabajo presenta un modelo econométrico de regresión lineal uniecuacional múltiple, para representar la relación económica que existe entre el precio spot de la energía eléctrica y algunas de las variables fundamentales del mercado de energía en Colombia, específicamente los caudales, dadas las características hidrodominadas del sistema de generación colombiano, y otras como la disponibilidad del Sistema Interconectado Nacional -SIN-, los precios de los contratos y el índice de red. Se evidenció a través de dos modelos que aunque la oferta, representada por el caudal, es altamente dependiente de la hidrología, no es suficiente como variable explicativa del precio, razón por la cual es necesario incluir otras variables. El modelo econométrico demuestra que además de los caudales, los precios de los contratos de largo plazo son significativos como variable explicativa del precio spot de la electricidad, además de estar positivamente correlacionados, mientras que los aportes de caudal y disponibilidad del sistema están negativamente correlacionados con el precio spot. Finalmente el modelo estimado puede ser utilizado para realizar pronósticos de corto y mediano plazo sobre el precio de la electricidad en bolsa de manera que permita a los generadores tomar decisiones operativas o de cobertura. Este artículo se constituye en un aporte a la investigación científica y tecnológica, y amplía de manera práctica el uso de la econometría en los mercados de electricidad.

Palabras clave: Precio spot de la electricidad, caudales, modelo de regresión lineal uniecuacional

Estudiante Maestría en Finanzas, Universidad de Medellín (2012); Especialista en Finanzas y Mercado de Capitales, Universidad de Medellín (2006); Contadora, Universidad de Antioquia (1998); Profesional Financiero, Dirección Finanzas Institucionales, Empresas Públicas de Medellín E.S.P. Este trabajo de investigación es realizado como trabajo de grado de la Maestría en Finanzas de la Universidad de Medellín, por la estudiante María del Carmen Quintero y bajo la dirección de Felipe Isaza. E-mail: maria.quintero.q@epm.com.co.

* Candidato a Doctor en Ingeniería-Industria y Organizaciones, Universidad Nacional de Colombia. Máster en Ingeniería Administrativa, Universidad Nacional de Colombia 2009., Ingeniero Mecánico Universidad Nacional de Colombia, 2006. Docente Tiempo completo Ingeniería Financiera. Investigador Grupo de Investigación en Ingeniería Financiera - GINIF y Grupo de Investigación en Energía. E-mail: fisaza@udem.edu.co 


\title{
HYDROLOGICAL AND REGULATORY DEPENDENCE ON THE FORMATION OF THE ENERGY PRICE IN A HYDRO-DOMINATED ENERGY SYSTEM: COLOMBIAN ELECTRICAL SYSTEM CASE
}

\begin{abstract}
This research presents a linear regression single-equation econometric model that represents the economic relationship between the electricity spot price and some fundamentals of the Colombian energy market, specifically hydro inflows, as it is a highly hydro-dominated system, and variables such as the system's generation availability index, bilateral long term contract prices, and the ratio between market's out of merit generation (positive reconciliation) and the actual real generation. The econometric model shows that hydro inflows as an explanatory variable is not sufficient to explain the spot price of electricity, and in consequence is necessary to use fundamentals as complementary explanatory variables. Results show besides hydro inflows, bilateral long term contract prices are positive and strong correlated with the spot price of electricity and acts as a significant explanatory variable, while hydro inflows and system's generation availability are negative correlated with the spot price of electricity. Finally the proposed model can be used for short and mid-term spot price forecasting in the Colombian energy market, in support of the decision making process of market agents. This article is a contribution to the scientific and technological research, and practically extends the applicability of econometric in finance.
\end{abstract}

Key words: Electricity spot price, water inflows, single equation linear regression models. 


\section{INTRODUCCIÓN}

La generación, en el mercado eléctrico colombiano, siempre se ha caracterizado por ser principalmente hidrodominada. Debido a la abundancia del recurso hídrico y a la topografía colombiana, la energía hidráulica ha sido desde siempre la fuente más atractiva para atender la demanda de energía eléctrica en Colombia.

Con las dificultades vividas en el pasado por la falta de expansión del sistema y la volatilidad en el precio de la energía eléctrica por la dependencia hidráulica, en especial en épocas de grandes sequías en Colombia, se ha visto la necesidad de diversificar las tecnologías de los recursos para la generación eléctrica. Al finalizar el año 2012 la capacidad hidráulica instalada representaba el $64 \%$ de los 14,361 MW totales instalados [1]; continúa en expansión el desarrollo de proyectos de esta tecnología, tanto de gran tamaño como de plantas menores. Por la alta participación que tiene el recurso hídrico en la conformación del sistema eléctrico colombiano existe una relación directa entre el precio de la energía y el nivel de caudales de los ríos que surten las plantas de generación. Se presume una alta dependencia de las fluctuaciones del precio a las variaciones de los caudales; como se expone en este artículo, el precio de la energía también es determinado por otras variables. Para explicar un precio en cualquier mercado se deben tener en cuenta la oferta, la demanda y los factores propios del mercado; para el caso de la energía, por el lado de la oferta están el recurso hidráulico que es la mayor fuente y el precio de los combustibles, los cuales, aunque sean marginales, son un insumo y hacen ver el costo de oportunidad de los hidráulicos; dentro de la oferta también se consideran la capacidad instalada del sistema interconectado nacional -SIN- y su disponibilidad; por el lado de la demanda, cuando hay incrementos fuertes, el precio tiende a subir. En Colombia hay una situación especial con el fenómeno de El Niño donde se afectan tanto la oferta como la demanda; la oferta porque hay menores lluvias y por tanto se incurre en costos adicionales, y por el lado de la demanda, porque a raíz del calor se aumentan los consumos de equipos de refrigeración, aires acondicionados, entre otros.

El precio spot de la energía eléctrica es determinado diariamente con base en el comportamiento de la oferta y la demanda, y corresponde al precio de oferta del recurso marginal no inflexible que se obtienen del despacho ideal [2], despacho que se obtiene considerando la demanda esperada del mercado y la oferta declarada por los generadores sometidos a despacho central, además de considerar variables como restricciones técnicas, contratos bilaterales, disponibilidad de combustibles, nivel de los embalses, entre otras variables técnicas. A pesar de que el precio de bolsa es una variable exclusiva del sector eléctrico, se ve influenciada por variables del mismo sector y de otros sectores económicos, tales como precio y disponibilidad de los combustibles, crecimiento de la demanda eléctrica, tamaño de los contratos bilaterales de las empresas generadoras, crecimiento del PIB y, muy probablemente, alguno o algunos rezagos de la propia variable. Las grandes empresas del sector tienen programas o modelos complejos de aproximación a pronósticos de largo plazo como el Modelo de Planeamiento Operativo de Energía -MPODE- ${ }^{1}$, para las estimaciones de precios de bolsa de corto plazo se pueden utilizar, entre otros, modelos de series de tiempo tales como los modelos ARMA o ARIMA, que han tenido un importante éxito en los estudios financieros debido a su gran utilidad en proyecciones razonables y creación de escenarios confiables en el corto plazo. Con el fin de comprender la formación del precio de bolsa es recomendable utilizar un modelo econométrico de regresión lineal múltiple que considere las variables fundamentales del mercado; de esta forma se puede tener más información sobre la reacción del merca-

\footnotetext{
Mediante la programación dual dinámica estocástica, permite el modelamiento de las diferentes variables fundamentales del mercado eléctrico colombiano (oferta, demanda, hidrología, costos y disponibilidad de combustibles, entre otras). Es utilizado por el operador del mercado XM S.A.E.S.P
} 
do frente a cambios en las variables determinantes del precio. Así se pueden complementar los análisis para la fijación y expectativa de precios en el mediano plazo, lo que sería de utilidad para los agentes de pequeña y gran capacidad que tengan interés en analizar tendencias en el comportamiento del precio de la electricidad, los análisis de mediano plazo del administrador del mercado y los seguimientos que hace la Superintendencia de Servicios Públicos Domiciliarios -SSPD.

Con la estimación de un modelo econométrico, se busca concluir cómo es la relación económica entre el precio spot de la energía en Colombia y las variables más significativas del sector como lo es el nivel de los caudales del SIN y otras variables económicas que, empíricamente y por las condiciones de este mercado, se presume tienen un nivel de significancia en la fluctuación del precio. El procesamiento de los datos y las estimaciones se efectúan aplicando el programa Eviews 6. El documento está estructurado de la siguiente manera; en la sección 2 se presenta el marco teórico y la revisión de literatura; en la sección 3, se presentan la especificación y la estimación del modelo econométrico, y en la sección 4, se presentan las conclusiones y la bibliografía.

\section{MARCOTEÓRICOY REVISIÓN DE LITERATURA}

Desde principios de los años ochenta, cuando se comenzaba a analizar la energía eléctrica como un commodity [3], y principalmente desde la reestructuración mundial de los mercados eléctricos hacia mercados de libre competencia, surgió la posibilidad de diseñar productos derivados (futuros y opciones sobre energía eléctrica) con fines de negociación y cobertura. La necesidad de pronosticar y modelar los precios de mercado para la toma de decisiones genera la oportunidad para diseñar modelos estadísticos adecuados que representen de la mejor manera el comportamiento del mercado abarcando las variables más significativas que afec- tan el precio de la energía eléctrica. Con base en lo anterior, se encuentran múltiples trabajos en la literatura científica donde se aborda el análisis, pronóstico y modelación de los precios de la energía; se reconocen inicialmente en la modelación algunas características particulares del precio de la energía eléctrica como los son la estacionalidad (determinada principalmente por factores climatológicos que afectan la oferta y demanda), la reversión a la media, la alta volatilidad y la ocurrencia de saltos como consecuencia del desbalance entre la oferta y la demanda en momentos específicos del tiempo [4, 5]. La mayoría de los trabajos buscan modelos que representen los comportamientos de la energía eléctrica teniendo en cuenta los elementos anteriores, como el trabajo presentado por Lucía y Schwartz [6] donde analizan los patrones estacionales que afectan los pronósticos de precios de energía y la forma como inciden en la valoración de derivados sobre energía eléctrica en una aplicación para el Nord Pool. Por su parte, Bhanot [7] utiliza variables dummy para el desarrollo de una función por tramos que capture los componentes estacionales mensuales y semanales, diferenciando los días de semana laboral y los fines de semana, mientras Weron [8] utiliza un modelo similar para la modelación del precio de energía con fines de valoración de opciones asiáticas para el Nord Pool, para fines de pronóstico y valoración de opciones. Para el pronóstico de corto plazo, Contreras, Espínola, Nogales \& Conejo [9] y Zhou, Yan, Ni, Li \& Nie [10] utilizan modelos de promedio móvil auto-regresivos ARMA y ARIMA. En Weron, [11] se propone un modelo de pronóstico de precio analizando la serie de tiempo mediante descomposición de Fourier. Para el caso colombiano se pueden resaltar los trabajos desarrollados por Botero y Cano [12], quienes presentan una metodología para analizar modelos de regresión sobre las series de tiempo de precios de energía eléctrica, identificando las relaciones entre el precio de la electricidad en Colombia y las acciones de intervención del regulador, las cuales fueron incorporadas como variables del modelo; 
en Gil Maya [13], con base en los desarrollos presentados por Schwartz, Lucia (2002) presenta un modelo para el análisis del valor y volatilidad del precio de la energía eléctrica en Colombia incorporando variables como la estacionalidad, los días de la semana y el efecto que tiene el fenómeno de El Niño. Grajales [14] analiza mediante un modelo de inferencia neurodifuso (ANFIS) la modelación del precio de la energía eléctrica, ajustado, de manera significativa, para el caso de la electricidad en bolsa en Colombia.

Como característica general de los modelos anteriores, estos trabajan directamente sobre las series de tiempo, analizando los factores estacionales directamente sobre el valor histórico del precio de la energía eléctrica, pues suponen que este incorpora directamente su efecto. Este trabajo se desarrolla con base en un modelo econométrico de regresión lineal uniecuacional multivariado con términos autorregresivos, que busca establecer la relación económica del precio de la energía en el mercado de electricidad colombiano como variable endógena, y los fundamentales del mismo mercado: oferta, demanda y disponibilidad del sistema como variables exógenas o explicativas. Se diferencia de otros estudios porque centra su atención en los fundamentales del mercado eléctrico colombiano para explicar la reacción del mercado a las principales variables que inducen el precio y no se centra solamente en las variables climatológicas. Se puede utilizar con fines de análisis del comportamiento del mercado y pronósticos de mediano plazo (semanas o meses).

Los modelos de regresión lineal uniecuacionales, se encuentran muy bien definidos y documentados en la literatura sobre econometría. Gujarati [15] es uno de los autores que más ha trabajado sobre este tipo de modelos. En estos una variable llamada dependiente es expresada como función lineal de una o más variables explicativas y se supone implícitamente que existen relaciones causales entre las variables y que van en una sola dirección: de las variables explicativas hacia la dependiente. Cuando se analiza la dependencia de una variable en una única variable explicativa, se define como modelo de regresión simple o con dos variables, y cuando se tiene más de una variable explicativa, se define como modelo de regresión múltiple, que finalmente es una extensión lógica del primer modelo.

La expresión lineal que define lo anterior es

$$
\begin{aligned}
& Y_{i}=\beta_{1}+\beta_{2} X_{i}+\mu_{i} \\
& Y_{i}=\beta_{0}+\beta_{1} X_{1}+\beta_{2} X_{2}+\beta_{3} X_{3}+\beta_{k} X_{k}+\mu_{i}
\end{aligned}
$$

\section{Donde:}

$Y_{i}$ es la variable dependiente

$X_{1}$ hasta $X_{k}$ son las variables explicativas

Los $\beta_{i}$ son coeficientes de regresión y el término $\mu_{i}$ es el término de error estocástico, en el cual se reúnen todas las variables que son omitidas en el modelo pero que afectan a $Y_{i}$.

El método de estimación utilizado en el modelo fue el de mínimos cuadrados ordinarios, que consiste en hacer mínima la suma de los cuadrados de los residuales, y trabaja bajo los siguientes supuestos:

1. El valor promedio de los errores es igual a cero.

2. No existe autocorrelación entre los errores, por lo tanto, la covarianza de los errores es igual a cero, lo cual implica que no existe autocorrelación en la variable dependiente.

3. La varianza de los errores es constante, es decir, existe la homocedasticidad.

4. No existe correlación entre los errores y las variables explicativas.

Para la especificación y estimación del modelo se utilizó la metodología Box Jenkins [16] que tiene un amplio uso en la modelación econométrica.

\section{ESTIMACIÓN MODELO ECONOMÉTRICO}

Para la estimación del modelo de regresión lineal simple, se tomó como variable dependiente el promedio mensual del precio spot de la energía en bol- 
sa, expresado en $\$ / \mathrm{kWh}$-(PBOLSA), y como variable independiente, el caudal promedio de los principales ríos del SIN, expresado en $\mathrm{m}^{3} / \mathrm{s}$-(CAUDAL). Las series están compuestas con datos promedio mensuales y se consideró el período enero de 2001 a diciembre de 2010; la información de ambas series proviene de XM S. A. ESP (operador del sistema interconectado nacional colombiano). [17]

Cada serie se analiza de forma individual, determinando patrones de comportamiento tales como tendencia, ciclicidad, estacionalidad y varianza. En la figura 1 se presentan las series de manera comparativa; en la serie caudal, los datos se encuentran alrededor de una media por lo que no muestran tendencia, contrario a la serie precio en la cual se observa una tendencia constante hasta finales del año 2007; a partir de este momento, se observa una tendencia de crecimiento relativamente constante. En el análisis de la figura 1 de ambas series se observa también la presencia de ciclicidad, explicada principalmente por los ciclos del océano Pacífico, evidenciado por un menor caudal promedio en los ríos del SIN durante 2003, 2006 y 2010 que corresponde con el calentamiento de la superficie del océano Pacífico; la estacionalidad se explica por los ciclos climáticos en el territorio co-

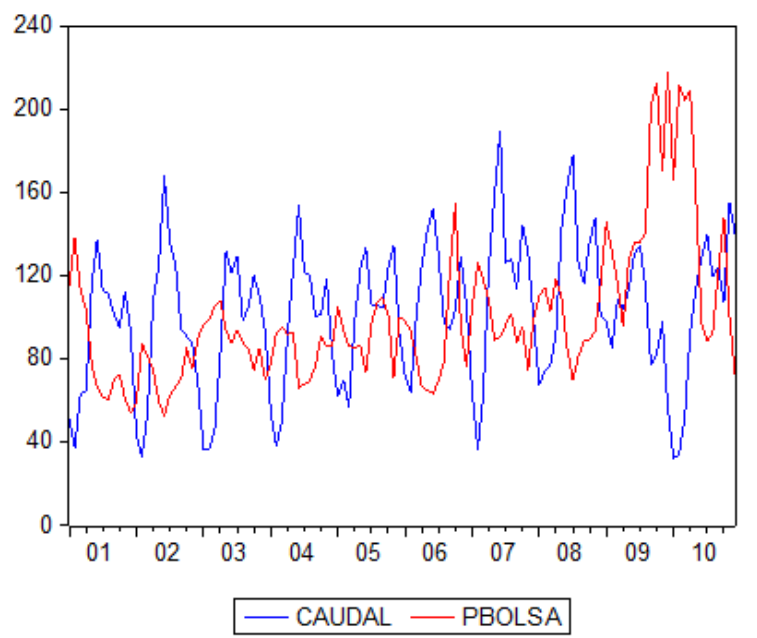

Figura 1: Comparación gráfica precio de bolsa-caudal Fuente: Elaboración propia en programa Eviews 6 lombiano; en términos generales existe una época de mayor hidrología en el segundo semestre de cada año. En general los procesos hidrológicos registran una componente estocástica importante que se encuentra relacionada de forma muy directa con los fenómenos de precipitación; para el precio de bolsa, teóricamente, se podría definir que es una serie estacional, coincidiendo con lo señalado por Pilipovic [4] y Clewlow, Strickland [5]; en períodos de baja hidrología los precios son más elevados y viceversa; normalmente en los meses de sequía el precio promedio es mucho más volátil; esto se observa a finales del año 2009 en donde a raíz del inicio del fenómeno de El Niño, el mercado de la energía colombiano fue intervenido por el ente regulador tratando de cubrir totalmente el riesgo de racionamiento.

Según [18], la mayoría de los procesos estudiados en hidrología no son estacionarios debido a que la función de distribución de probabilidad que describe la población cambia con el tiempo. Utilizando el test Dickey Fuller aumentado se acepta la hipótesis nula de que ambas series tienen raíz unitaria por lo que requieren de una primera diferenciación para lograr estacionariedad; se aplica también la transformación logarítmica a las series diferenciadas para controlar los problemas de heterocedasticidad que afecten la especificación del modelo econométrico. A partir de las dos series ya transformadas, se inicia la estimación del modelo que mejor represente la relación de las variables, considerando como supuesto que aunque el precio de la energía no solo es determinado por la hidrología, sí es la variable de mayor incidencia en la determinación y la proyección del precio a corto plazo.

En una expresión lineal el precio de bolsa en función del caudal se presenta así:

$$
\text { Pbolsa }_{t}=c+B * \text { Caudal }_{t}+\varepsilon_{t}
$$

Donde:

Pbols $_{\mathrm{t}}$ es el precio spot de la energía en bolsa $c$ es el término independiente 
$\beta$ es la elasticidad entre precio y caudal (series en diferencias logarítmicas)

Caudal es el caudal

$\varepsilon_{\mathrm{t}}$ es la perturbación aleatoria.

Se incluye también una variable tipo dummy dicotómica² (INTERVENC) que representa el impacto súbito que generó sobre el precio el hecho de que el Gobierno decretara oficialmente la presencia del fenómeno del El Niño a finales del año 2006.

Se hace el análisis de los criterios que ayudan a validar que no existan problemas de autocorrelación serial y determinar el comportamiento de los residuales a través de los correlogramas; lo que se busca es un mecanismo autorregresivo (AR) que los explique o un proceso de medias móviles (MA) que lo represente. En la tabla 1 se observan los resultados de la estimación del modelo en donde, además de la variable caudal como variable explicativa, se incluyen los términos autorregresivos AR(2) y AR(3) que presentan una buena correlación con la propia serie precio de bolsa.

El coeficiente de la variable independiente es negativo lo que confirma la relación inversa entre las variables; por cada unidad porcentual que aumenta el caudal, el precio de la energía disminuye en $-0,26$. Esta situación se confirma con el cálculo del coeficiente de correlación de $-0,36$ donde se valida la correlación negativa entre el precio de bolsa y los caudales.

En otros resultados que arroja el modelo se aprecia el coeficiente de determinación $\left(\mathrm{R}^{2}\right)$ con un valor de 0.28 , que indica la proporción de variación total explicada mediante la relación lineal entre el caudal y el precio de bolsa; este coeficiente es relativamente bajo, y se puede concluir que la perturbación aleatoria está asumiendo todo lo que el caudal no puede explicar; por lo tanto, se deben incluir otras variables fundamentales del mercado, de manera que ||t sea efectivamente ruido blanco.

2 Variable que toma valor 1 en el período especificado, cuando una alerta del gobierno hizo que los agentes incrementaran el precio por una percepción de riesgo. En otros casos esta variable toma valor cero.
En la figura 2, se presentan los residuales del modelo. Los crecimientos que presentan la serie precio de bolsa no los puede explicar el modelo y por eso las desviaciones son tan altas que superan el nivel de confianza del $95 \%$.

La primera conclusión después de tratar de realizar la estimación es que existe una correlación significativa entre las variables analizadas pero el modelo no demuestra que el precio de bolsa sea explicado completamente por la variable caudal y sus propios rezagos, por lo que es necesario incluir algunas variables fundamentales del mercado que

Tabla 1: Resultados estimación Modelo regresión lineal simple

\begin{tabular}{|l|c|c|c|}
\hline \multicolumn{4}{|c|}{ Variable Dependiente: DLOG(PBOLSA) } \\
\hline \multicolumn{1}{|c|}{ Variable } & Coeficiente & Estadistico & P-valor \\
\hline C & $-0,004522$ & $-0,465072$ & 0,6428 \\
\hline DLOG(CAUDAL) & $-0,266572$ & $-4,931,493$ & 0 \\
\hline INTERVENC & 0,175373 & $2,220,357$ & 0,0284 \\
\hline AR(2) & $-0,17655$ & $-1,867,092$ & 0,0645 \\
\hline AR(3) & $-0,325949$ & $-351,056$ & 0,0006 \\
\hline R2 ajustado & 0,281137 & & \\
\hline Coef. Correlación & $-0,363967$ & & \\
\hline
\end{tabular}

Fuente: Elaboración propia en programa Eviews 6

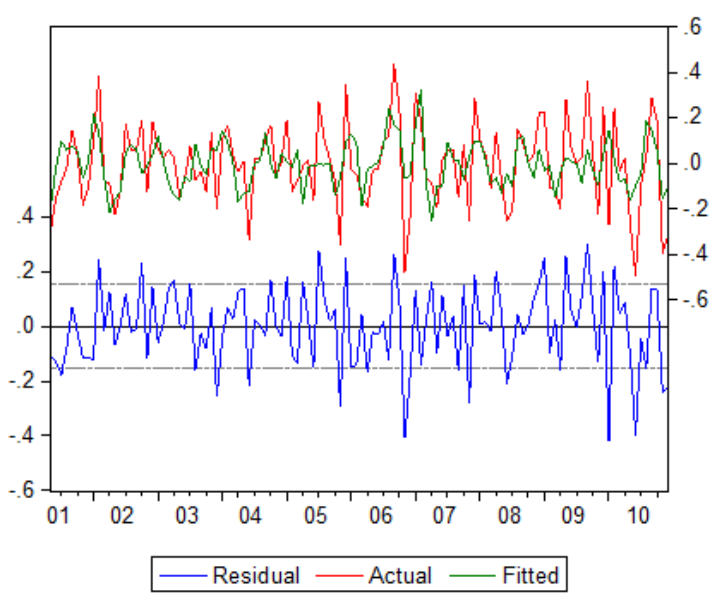

Figura 2: Residuales Modelo regresión lineal simple Fuente: Elaboración propia en programa Eviews 6 
representen la oferta y la demanda de energía como variables independientes y así

La segunda estimación surge como consecuencia de los resultados de modelos anterior y se fundamenta en un modelo multivariado, seleccionando algunas variables representativas del Mercado Eléctrico Colombiano -MEM- variables como: capacidad efectiva neta, demanda del SIN y restricciones fueron excluidas, ya que al determinar su grado de diferenciación e incorporarlas al modelo, se detectó que algunas de estas estaban correlacionadas, lo que obligó a excluirlas para evitar que se alterara artificialmente la bondad de ajuste, es decir, evitar la multicolinealidad.

Además de la variable caudal, las otras variables independientes seleccionadas fueron:

Disponibilidad total promedio que mensualmente se obtiene en el SIN-(DISPSIN) expresada en MW (figura 3a); se incluyó porque teóricamente se espera que entre menos capacidad disponible presente el sistema, el precio de bolsa aumente y viceversa; Precio de los contratos-(PCONT) expresado en $\$ / \mathrm{kWh}$ (figura $3 \mathrm{~b}$ ), esta variable fue incluida en el análisis porque puede reflejar el interés de los generadores por atender la demanda para cumplir con los contratos pactados sin tener que comprar en la bolsa de energía. Lo anterior significa que los agentes procuran aumentar el precio de bolsa cuando tienen contratos bajos, y participar con mucha generación cuando tienen alta contratación. Indice de red-(IRED) establece la relación entre la generación fuera de mérito (reconciliación positiva) y la generación real del SIN que aumenta proporcionalmente con las restricciones del sistema (figura 3c); se incluyó en el modelo porque cuando se han presentado indisponibilidades o restricciones en el Sistema de Transmisión Nacional, algunos de los recursos asignados para generar por mérito de precio se reemplazan por otros, lo que afecta el precio de bolsa. Las restricciones que perduran durante un período de tiempo considerable hacen que aumente el nivel de embalsamiento de los recursos hidráulicos que no pudieron entregar su energía y finalmente causa un menor precio de bolsa. La información de las series proviene de XM S. A. ESP, filial de ISA, y se considera el mismo periodo de tiempo que el caudal y el precio de bolsa.

En la figura 3 se presentan las series de manera individual y se determinan los patrones de comportamiento; para el caso de la disponibilidad del SIN, se observa una tendencia a la baja que se estabiliza al final del periodo, además de estacionalidad y no estacionariedad; en el precio de los contratos se concluye que no tiene una tendencia constante: en los dos primeros años del período analizado tuvo crecimiento para luego presentar una tendencia decreciente entre 2003-2006 y nuevamente mostrar una tendencia creciente entre los años 2007 y 2010, además de tener estacionalidad; al comienzo de cada año es cuando los precios de los contratos son más altos; esto es lógico pues normalmente los primeros meses del año son los que históricamente muestran menores precipitaciones fluviales y el clima seco tiende a elevar el precio de bolsa. El índice de red muestra valores altos al comienzo del período analizado, pero las inversiones en infraestructura y las intervenciones regulatorias han logrado disminuir las indisponibilidades del sistema; se observa una alta volatilidad en esta serie. Tanto la disponibilidad del SIN, como los precios de los contratos son series no estacionarias y requieren de una primera diferencia para lograr estacionariedad.

Al generar el test de los residuos, el término autorregresivo AR(5) tiene una probabilidad inferior a 0,05; se incorpora en la estimación del modelo y se obtiene el modelo definitivo; sus resultados se observan en la tabla 2.

$$
\begin{aligned}
\text { Pbolsa }_{t}= & c+B^{*} \text { Caudal }_{t}+\text { Dispsin }_{t}+\text { Pcont }_{t} \\
& + \text { Ired }_{t}+\text { Intervenc }_{t}+A R(5)+\varepsilon_{t}
\end{aligned}
$$

Este modelo autorregresivo multivariado tiene un índice $\mathrm{R}^{2}$ de 0,48 , el cual mejora comparado con el mismo índice del modelo univariado: se acerca 
DISPSIN

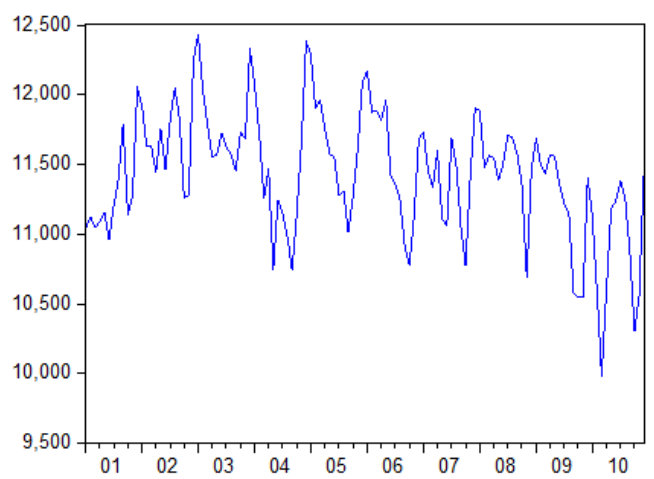

Figura 3 a)
PCONT

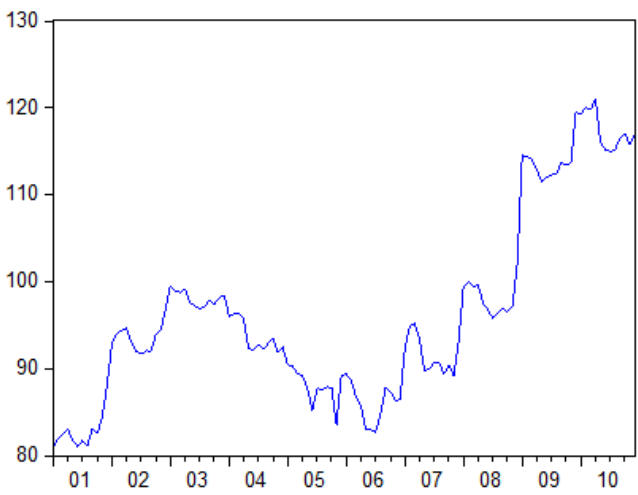

Figura 3 b)

IRED

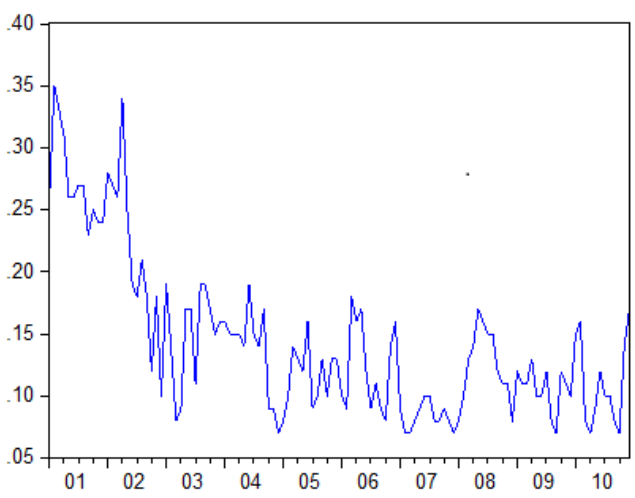

Figura $3 \mathrm{c}$ )

Figura 3: Series Modelo de Regresión Lineal Múltiple

Fuente: Elaboración propia en programa Eviews6

Tabla 2. Resultados estimación Modelo regresión lineal múltiple

\begin{tabular}{|c|c|c|c|c|}
\hline \multicolumn{3}{|c|}{ Variable Dependiente: DLOG(PBOLSA) } & \multirow[b]{2}{*}{ P-valor } & \multirow[b]{2}{*}{ Coef. Correlación } \\
\hline Variable & Coeficiente & Estadístico & & \\
\hline $\mathrm{C}$ & 0,096786 & $2,348,392$ & 0,0207 & \\
\hline DLOG(CAUDAL) & $-0,113465$ & $-2,495,885$ & 0,0141 & $-0,363967$ \\
\hline DLOG(DISPSIN) & $-2,083,604$ & $-5,689,623$ & 0 & $-0,498581$ \\
\hline DLOG(PCONT) & $3,555,685$ & $6,131,768$ & 0 & 0,661089 \\
\hline IRED & $-0,827185$ & $-2,958,379$ & 0,0038 & $-0,372359$ \\
\hline INTERVENC & 0,139053 & $1,911,304$ & 0,0586 & \\
\hline $\operatorname{AR}(5)$ & 0,315037 & 330,491 & 0,0013 & \\
\hline R2 ajustado & 0,486955 & & & \\
\hline
\end{tabular}

Fuente: Elaboración propia en programa Eviews6 
más a 1; las raíces unitarias de los términos autorregresivos $\mathrm{AR}$ son menores a 1 por lo que no se está sobrediferenciando ninguna de las variables; el test de raíces unitarias sobre los residuos confirma que el modelo cointegra, es decir, que los diferentes grados de integración de las variables no afectan los supuestos del modelo lineal.

Las pruebas sobre los residuales permiten aceptar la condición de homocedasticidad ${ }^{3}$; se tiene, además, que los residuos son incorrelacionados en el tiempo lo que es una condición necesaria para este tipo de modelos; se verificó mediante la prueba Jarque Bera, la cual con una probabilidad de 0,82 permite aceptar la hipótesis de que los residuales siguen una distribución normal.

Como se puede observar en la figura 4 , se verificó que la representación gráfica de los residuos sí corresponde a la de un ruido blanco, con lo que el ajuste del modelo estimado es bueno en el corto plazo, no obstante la alta volatilidad de la serie de precio de bolsa. Finalmente se verificó que la representación gráfica de los residuos si corresponde a la de un ruido blanco.

\section{CONCLUSIONES}

Analizando la relación entre las variables del modelo y el precio de bolsa desde el punto de vista de coeficientes de correlación de las variables de la regresión, se concluye que el precio de la energía está fuerte y directamente correlacionado con el precio de los contratos (correlación 0.6610 ), puesto que estos reflejan las expectativas de los agentes contratantes y oferentes del mercado, teniendo en cuenta las variables de disponibilidad y caudal, las cuales, como se observa y era de esperarse, presentan una correlación negativa, que refleja un comportamiento inverso, lo que verifica la dependencia de la disponibilidad hídrica, en términos de aportes de caudal y disponibilidad de embalse (correlaciones de -0.3639 y -0.4985 , respectivamente), pues a menor expectativa de disponibilidad y aportes,

Test autocorrelograma de los residuales al cuadrado con probabilidad de t mayor a 0.05

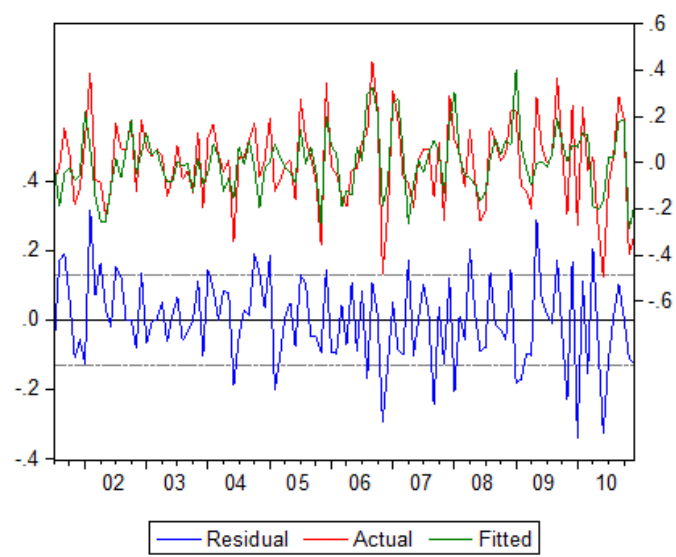

Figura 4. Residuales Modelo regresión lineal múltiple Fuente: Elaboración propia en programa Eviews6

mayor será el costo de marginal y en consecuencia el precio de bolsa

Mediante la especificación del modelo econométrico multivariado se puede concluir que la formación del precio de bolsa de la energía eléctrica en el mercado colombiano obedece significativamente a los fundamentales del mercado: oferta (disponibilidad de la red, caudales y capacidad instalada del sistema) y variaciones de la demanda. Los precios de los contratos de largo plazo resultaron significativos en la especificación del modelo, y de esta forma se puede inferir que la señal de largo plazo equilibra el precio spot de la electricidad, evitando que los generadores tengan incentivos para subir el precio deliberadamente cuando tienen que comprar en el spot las cantidades de energía que comprometieron a largo plazo y que no son cubiertas totalmente por su propia generación.

El mercado eléctrico colombiano es bastante complejo; con el modelo econométrico se vislumbran los determinantes fundamentales del precio. No obstante, con el fin de tener análisis más detallados de largo plazo, más precisos sobre las condiciones del mercado, es recomendable utilizar modelos de planeamiento operativo que, además de incorporar información de la oferta y la demanda de energía, permitan incluir en el análisis otras variables como el cargo por confiabilidad que se 
paga para asegurar la inversión en la oferta a largo plazo, los costos y restricciones de combustibles, la disponibilidad de la red de transporte, la interconexión con otros países y modelar la volatilidad hidrológica con herramientas más eficaces, entre otros.

Como propuesta de trabajo para investigaciones posteriores, se podría aprovechar el modelo desarrollado para la valoración de instrumentos financieros que permita incorporar en la valoración de futuros y opciones de electricidad la relación entre los aportes hidrológicos y el precio de la energía eléctrica en un sistema hidrodominado.

\section{REFERENCIAS}

[1] XM EXPERTOS EN MERCADO, "Informe de Operación del SIN y administración del mercado 2012,” 2012.

[2] UNIDAD DE PLANEACIÓN MINERO ENERGÉTICA, "Una visión del mercado eléctrico colombiano," 2004.

[3] T. W. Berrie, y M. Hoyle, "Treating energy as a commodity,” Energy Policy, vol. 13, pp. 506-510, 1985.

[4] D. Pilipovic, Energy Risk: Valuing and Managing Energy Derivatives. New York: McGraw-Hill, 2007.

[5] L. Clewlow, y C. Strickland, Energy Derivatives: Pricing $\mathbb{E}$ Risk Management New York: Lacima Publications, 2000.

[6] Julio L. Lucía, y Eduardo S. Schwarts, "Electricity prices and power derivatives: Evidence from the Nordic Power Exchange," 2000.

[7] K. Bhanot, "Value of an option to purchase electric power - the case of uncertain consumption "Elsevier, vol. 24, pp. 121-137, 2002.
[8] R. Weron, "Market price of risk implied by Asian-style electricity options and futures," Elsevier, vol. 30, pp. 1098-1115, 2008.

[9] J. Contreras, R. Espínola, F J. Nogales, A. J. Conejo "ARIMA Models to Predict Next-Day Electricity Prices “ IEEE Treansaction on Power Systems, pp. 1014-1020, 2003.

[10] Z. N. Yan, Y. X. ; Li, G. ; Nie, Y. , "Electricity price forecasting with confidence-interval estimation through an extended ARIMA approach "IEEE Procedings - Generation, Transmision and Distribution, pp. 187-195, 2006.

[11] R. Weron, M. Bierbrauerb, S. Truck, "Modeling electricity prices: jump di\%usion andregime switching," Elsevier, pp. 39-48, 2003.

[12] S. Botero Botero, J. Alfonso Cano Cano, "Análisis de series de tiempo para la predicción de los precios de la energía en la bolsa de Colombia”. Cuadernos de Economía, 2008.

[13] M. Gil Zapata, C. Maya Ochoa, "Modelación de la volatilidad de los precios de la energía eléctrica en Colombia," Revista Ingenierias, Universidad de Medellin, vol. 7, 2008.

[14] D. G. Bedoya, "Modelación del precio de la energía en Colombia usando un modelo de inferencia neurodifuso (anfis )," Revista Soluciones de Posgrado EIA, pp. 25-38, 2009.

[15] D. Gujarati, Econometría. Estados Unidos, 2004.

[16] G. M. J. G. E. P Box, Time Series Analisis: Forescasting and Control: Holden Day 1970.

[17] “XM S. A. ESP," in Informe de Administración y Operación del Mercado 2011, ed, 2012.

[18] J. S. Arenas Cárdenas, Desarrollo de un modelo de pronóstico de caudales semalaes asociado a la variabilidad climática intranual en Colombia. Medellín, Antioquia, Colombia, 2009. 
